\title{
26431 - EFFECT OF GENERAL ANESTHESIA ON BIVENTRICULAR DIASTOLIC FUNCTION
}

\section{Pierre Couture MD FRCPC, André Y Denault, MD FRCPC; Yanfen Shi, MD; Michel Pellerin, Jean-Claude Tardif, MD FRCPC; Montreal Heart Institution, Montreal, QUEBEC, Canada}

INTRODUCTION: Patients with left ventricular (LV) diastolic dysfunction are at higher risk of hemodynamic instability after coronary artery bypass (CABG) grafting (1). However, the effect of induction of general anesthesia on right and left filling pattern is unknown and may influence the assessment of diastolic dysfunction (DD).

METHODS: After IRB approval, 50 patients with DD undergoing CABG were studied. Preoperative transthoracic echocardiographic examination was performed on the day before surgery. Transesophageal echocardiography (TEE) was used after the induction of general anesthesia (GA). The anesthesia protocol consisted in a combination of sufentanil, midazolam, isoflurane, and pancuronium. Pulsed wave (PW) Doppler of the transmitral and transtricuspid inflow (TMF and TTF, E and A wave), pulmonary and hepatic venous flow (PVF and HVF: S, D and Ar waves at atrial contraction) were measured. Mitral (Em, Am) and tricuspid annulus velocities (Et, At) were assessed by tissue Doppler imaging (TDI). Left ventricular end-diastolic area (EDA), end-systolic area (ESA), and fractional area change (FAC) from transgastric midpapillary view were measured. From the apical 4-chamber view, we measured the left atrium (LA) and right atrium (RA) diameters. Mean arterial pressure (MAP) and heart rate (HR) were noted. Student $t$ tests were used for comparison. $\mathrm{P}$ value $<0.05$ was considered significant. RESULTS: After GA, we observed a decrease in HR (66 \pm 12 vs 55 \pm 9 ; $\mathrm{p}<0.0001)$ while the MAP remains stable $(86.1 \pm 9.0$ vs $85.6 \pm 26.5 ; \mathrm{p}=0.94)$. We observed significant decreases $(\mathrm{P}<0.05)$ in TMF E and A waves, Em and Am, TTF E and A waves, and in Et and At. However, TMF and TTF E/A ratios, Em/Am and Et/At increased after GA, indicating a greater decrease in the atrial components of these ratios. We also observed an increase in LA and RA diameters. Most components of the HVF and PVF were decreased. However, the PVF and HVF S/D ratios increased. EDA and ESA decreased without change in FAC.

CONCLUSION: Bi-ventricular filling patterns are significantly altered after induction of GA, as reflected by a decrease in LV size, an increase in bi-atrial diameters, and a decrease in most components of TMF, TTF, PVF, and HVF.

REFERENCE: 1) Anesth Analg 2001;92:291-8. 